\title{
Regional geoelectrical complexity of the Western Canada Basin from magnetotelluric tensor invariants
}

\author{
F. W. Jones ${ }^{1}$, R. A. Munro ${ }^{1 *}$, J. A. Craven ${ }^{2}$, D. E. Boerner ${ }^{2}$, R. D. Kurtz ${ }^{2}$, and R. D. Sydora ${ }^{1}$ \\ ${ }^{1}$ Department of Physics, University of Alberta, Edmonton, Canada T6G $2 \mathrm{JI}$ \\ ${ }^{2}$ Geological Survey of Canada, 601 Booth Street, Ottawa, Canada K1A 0E8
}

(Received July 6, 2001; Revised June 5, 2002; Accepted July 29, 2002)

\begin{abstract}
Magnetotelluric data from 323 sites in the Western Canada Basin have provided impedance tensors from which invariants based on Mohr circle analysis are calculated. From thresholds determined from an initial examination of the data, the invariant responses are used to explore the dimensionalities of subsurface structures. This leads to a classification of the electrical structure of the region with respect to dimensionality wherein zones with sites of similar nature are delineated, and demonstrates that the invariants can be used as a dimensionality indicator to infer regional geoelectrical complexity. It is observed that the dimensionality appears to increase with period and this result is consistent with the response from sounding a zone of increasing areal extent and may indicate more laterally complex structure at depth, but may also be due to fundamental limitations of galvanic distortion analysis.
\end{abstract}

\section{Introduction}

From the magnetotelluric (MT) method, the electric and magnetic field components measured at the surface of the earth can be related through the tensor equation

$$
\left[\begin{array}{c}
E_{x} \\
E_{y}
\end{array}\right]=\left[\begin{array}{ll}
Z_{x x} & Z_{x y} \\
Z_{y x} & Z_{y y}
\end{array}\right] \cdot\left[\begin{array}{l}
H_{x} \\
H_{y}
\end{array}\right]
$$

where $E_{x}, E_{y}$ and $H_{x}, H_{y}$ represent horizontal electric and magnetic field components measured along two orthogonal axes. The elements $Z_{i j}$ comprise the MT impedance tensor and are generally complex. All quantities in (1) are frequency dependent.

The application of Mohr circle analysis to MT data has been proposed and used by Lilley $(1976,1993 \mathrm{a}, \mathrm{b}, \mathrm{c})$ as a means of displaying MT data and investigating the implied structural dimensionality and anisotropy. Weaver et al. (2000) characterized the MT tensor in terms of rotational invariants "... in such a way as to have clear representations on a Mohr circle diagram and also to reveal geoelectric properties of the Earth near the site where impedance data are measured."

In this work, we investigate the seven invariants defined by Weaver et al. (2000) for the 323 MT sites of the Alberta Lithoprobe Transect, and from them classify zones in the Western Canada Basin as to their structural dimensionality. The purpose of the work is two-fold. The invariants can provide insight into what physical properties are implied by the measured tensor, and the goal is to provide mathematical insight to a geological interpretation of a real data set. Weaver

\footnotetext{
*Now at CGG Canada Services Ltd., 700, 404 - 6th Avenue SW, Calgary, Canada T2P 0R9.
}

Copy right(c) The Society of Geomagnetism and Earth, Planetary and Space Sciences (SGEPSS); The Seismological Society of Japan; The Volcanological Society of Japan; The Geodetic Society of Japan; The Japanese Society for Planetary Sciences. et al. (2000) use a numerical evaluation of a synthetic model to find criteria or thresholds against which the invariants can be compared in order to determine the dimensionality of the electrical conductivity structure of the earth near a site. In this paper we attempt to refine the procedure for developing these criteria by using information from Bahr's (1991) analysis and Groom-Bailey (1989) decomposition of the data to calibrate the thresholds. Secondly, we use the calibrated thresholds to explore for new features in the Alberta data set, which by virtue of its large size, is difficult to visualize in a simple manner such as in map form.

\section{The Seven Invariants}

In the notation of Weaver et al. (2000), the matrix expression for the MT tensor is of the form

$$
M=\left(\begin{array}{l}
\xi_{1}+\xi_{3} \xi_{2}+\xi_{4} \\
\xi_{2}-\xi_{4} \xi_{1}-\xi_{3}
\end{array}\right)+i\left(\begin{array}{l}
\eta_{1}+\eta_{3} \eta_{2}+\eta_{4} \\
\eta_{2}-\eta_{4} \eta_{1}-\eta_{3}
\end{array}\right)
$$

and the seven invariants defined by them are:

$$
\begin{aligned}
& I_{1}=\left(\xi_{4}^{2}+\xi_{1}^{2}\right)^{1 / 2} \\
& I_{2}=\left(\eta_{4}^{2}+\eta_{1}^{2}\right)^{1 / 2} \\
& I_{3}=\frac{\left(\xi_{2}^{2}+\xi_{3}^{2}\right)^{1 / 2}}{I_{1}} \\
& I_{4}=\frac{\left(\eta_{2}^{2}+\eta_{3}^{2}\right)^{1 / 2}}{I_{2}} \\
& I_{5}=\frac{\xi_{4} \eta_{1}+\xi_{1} \eta_{4}}{I_{1} I_{2}} \\
& I_{6}=\frac{\xi_{4} \eta_{1}-\xi_{1} \eta_{4}}{I_{1} I_{2}} \\
& I_{7}=\frac{\left(d_{41}-d_{23}\right)}{Q}
\end{aligned}
$$




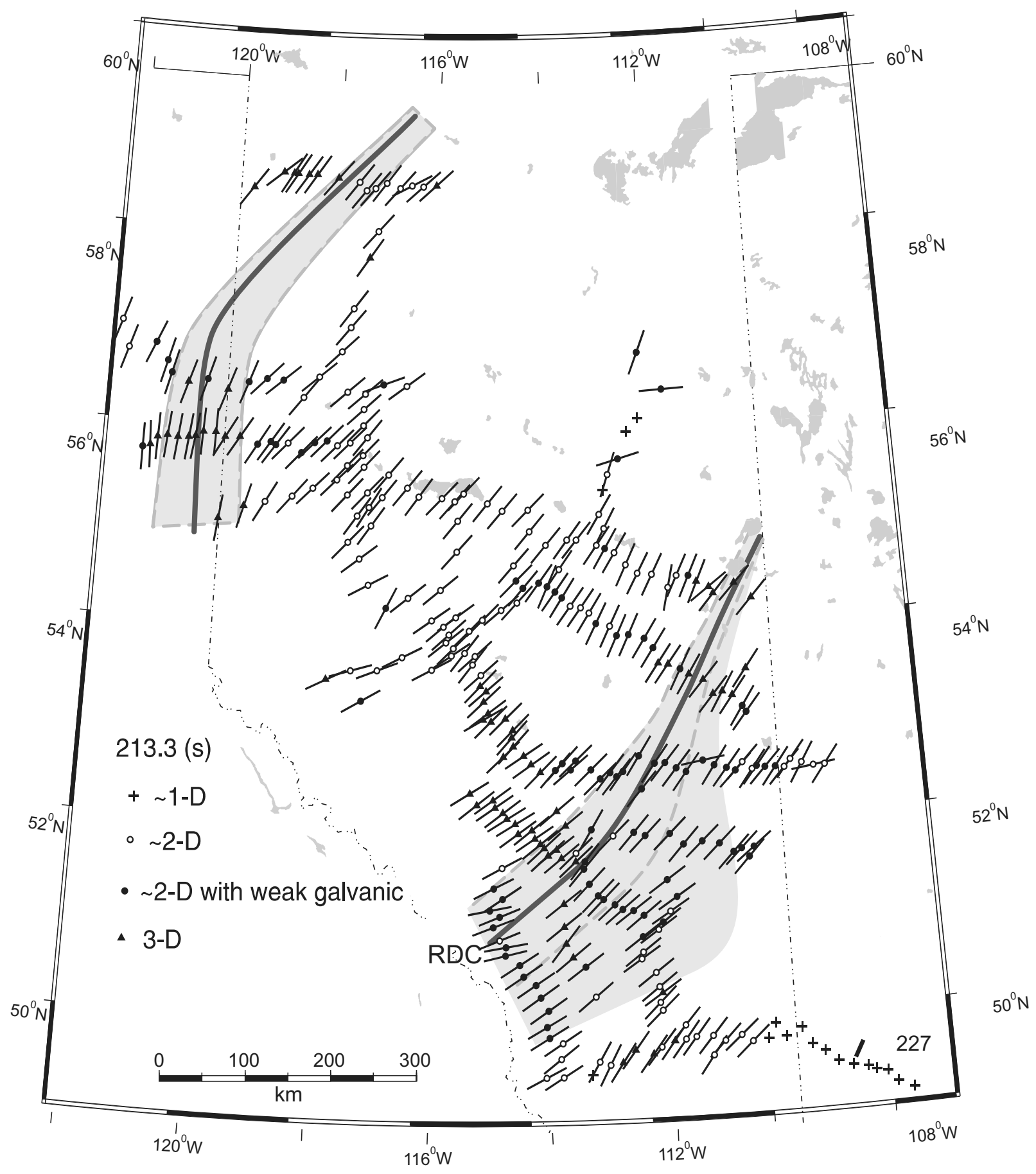

Fig. 1. The Province of Alberta with invariant classification for each site at $213.3 \mathrm{~s}$ period according to the dimensionality of the earth (1D, 2D, 2D with galvanic scattering and 3D). Geoelectric strike directions determined from the Groom-Bailey (1989) decomposition method for a period band around $300 \mathrm{~s}$ (see Boerner et al., 2000) are indicated for the 2D and 3D sites. The gray shading shows the region where the TE data indicate a strong conductor and the dashed lines indicate where the TE response of confirmed conductors is prominent (see Boerner et al., 2000). The heavy solid lines indicate the strikes of buried conductors where RDC $=$ Red Deer Conductor.

$$
\begin{aligned}
& \text { where } \quad d_{i j}=\frac{\xi_{i} \eta_{j}-\xi_{j} \eta_{i}}{\left(\xi_{4}^{2}+\xi_{1}^{2}\right)^{1 / 2}\left(\eta_{4}^{2}+\eta_{1}^{2}\right)^{1 / 2}} \\
& \text { and } Q=\left[\left(d_{12}-d_{34}\right)^{2}+\left(d_{13}+d_{24}\right)^{2}\right]^{1 / 2}
\end{aligned}
$$

The Weaver et al. (2000) $\xi_{i}$ and $\eta_{i}$ can be related to the more familiar impedance tensor elements. The classical expression for the tensor is $Z=\left[\begin{array}{cc}Z_{x x} & Z_{x y} \\ Z_{y x} & Z_{y y}\end{array}\right]$ where $Z=\operatorname{Re} Z+i \operatorname{Im} Z$. Hence

$$
\begin{aligned}
\xi_{1} & =\frac{1}{2}\left(Z_{x x}^{r}+Z_{y y}^{r}\right) & \eta_{1} & =\frac{1}{2}\left(Z_{x x}^{i}+Z_{y y}^{i}\right) \\
\xi_{2} & =\frac{1}{2}\left(Z_{x y}^{r}+Z_{y x}^{r}\right) & \eta_{2} & =\frac{1}{2}\left(Z_{x y}^{i}+Z_{y x}^{i}\right) \\
\xi_{3} & =\frac{1}{2}\left(Z_{x x}^{r}-Z_{y y}^{r}\right) & \eta_{3} & =\frac{1}{2}\left(Z_{x x}^{i}-Z_{y y}^{i}\right) \\
\xi_{4} & =\frac{1}{2}\left(Z_{x y}^{r}-Z_{y x}^{r}\right) & \eta_{4} & =\frac{1}{2}\left(Z_{x y}^{i}-Z_{y x}^{i}\right)
\end{aligned}
$$




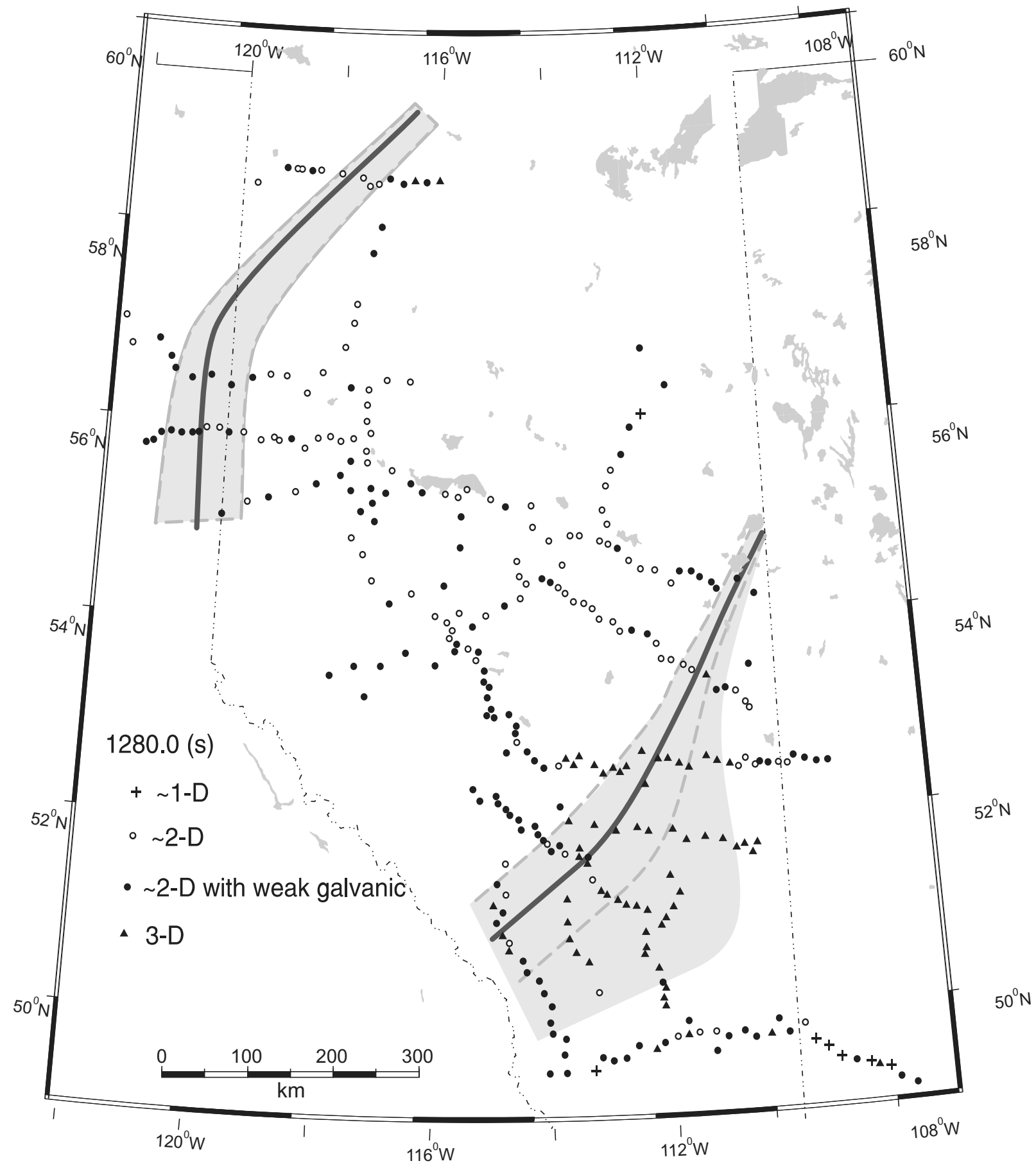

Fig. 2. As Fig. 1, but for $1280.0 \mathrm{~s}$ period.

We see from (10) that the first two invariants (Eqs. (3) and (4)) correspond to Lilley's (1993a) real and quadrature central impedances which are taken by Weaver et al. (2000) as the fundamental invariants for MT applications.

From (10) it is evident that, for example, $\xi_{1}, \xi_{2}$ and $\xi_{3}$ would be expected to be zero in a 1D situation, whereas $\xi_{4}$ would not and so $I_{1}$ and $I_{2}$ will be non-negligible but $I_{3}, I_{4}$, $I_{5}$ and $I_{6}$ will be negligible. Similarly, we see that for $2 \mathrm{D}$ structure $I_{3}$ and $I_{4}$ will be non-negligible since in this case $Z_{x y} \neq-Z_{y x}$ (axis along strike), or if neither axis is along strike $Z_{x x}=-Z_{y y} \neq 0$.

Weaver et al. (2000) discuss the physical interpretation of the invariants at length and give numerical calculations for a synthetic model to illustrate the implications of the various ranges of values of them. Briefly,

(1) If $I_{3} \sim I_{4} \sim I_{5} \sim I_{6} \sim 0$, (i.e. $I_{1}$ and $I_{2}$ non-negligible) then a $1 \mathrm{D}$ (i.e. horizontally layered) structure is implied.

(2) If $I_{5} \sim I_{6} \sim 0$ (with $I_{3}$ and $I_{4}$ non-negligible) and either $I_{7} \sim 0$ or $Q \sim 0$ then a $2 \mathrm{D}$ structure is implied.

(3) If $I_{6} \sim I_{7} \sim 0$, (all others non-negligible) then two possible distortions can exist (see Weaver et al., 2000).

(4) If $I_{7} \sim 0$ (all others non-negligible) then a small-scale 

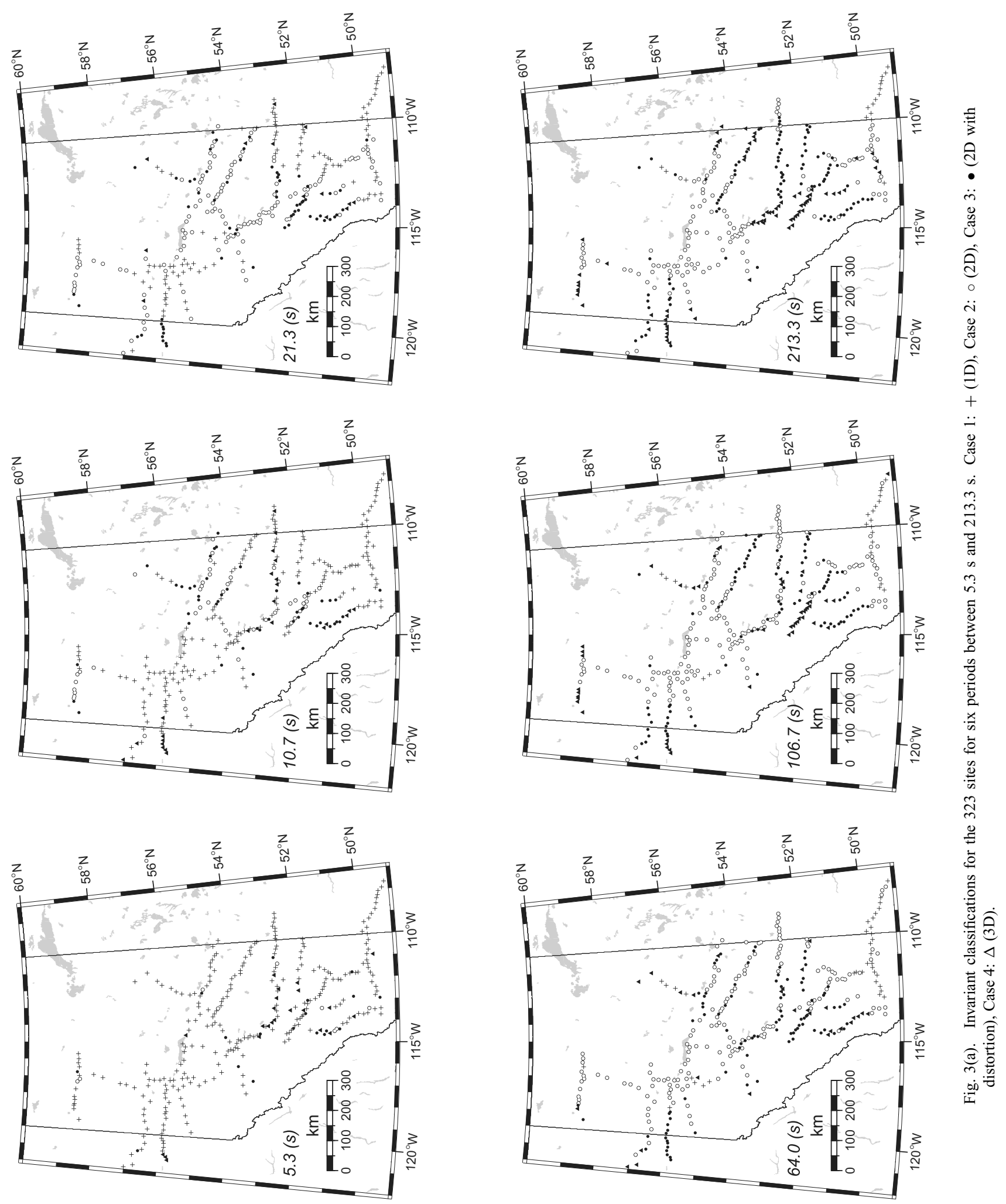

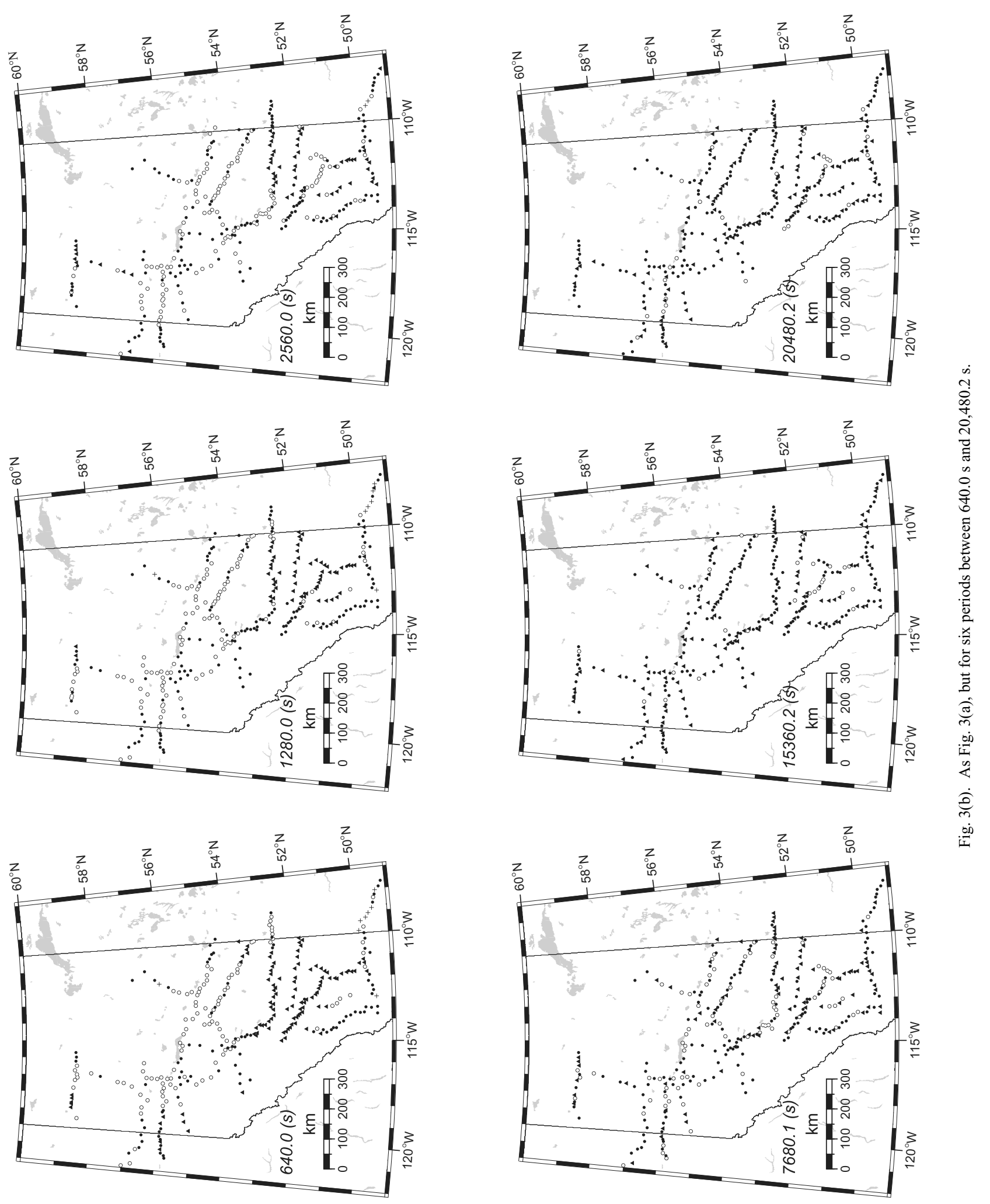
distortion in a 2D region is implied.

(5) If $I_{7}$ is non-negligible, then a 3D structure of regional scale is implied.

Therefore, depending on the relative values of these invariants, each site can be classified and zones for which the sites are similar can be delineated.

\section{Classification of Sites}

The Alberta sites were classified as follows:

Case 1: $I_{1}, I_{2}$ non-negligible (always true, Weaver et al., 2000), with all other invariants below the thresholds indicated in the following.

Case 2: Case 1 but with $I_{3}, I_{4}>$ (threshold 1), and either $I_{7}$ or $Q$ negligible (i.e. $<0.15$ which is in the range suggested by Weaver et al. (2000))

$$
\text { where }(\text { threshold } 1)=\frac{0.75}{\sqrt{\text { period }}}+0.1000 .
$$

Case 3: Case 2 but with $\left(I_{5}\right.$ or $\left.I_{6}\right)>($ threshold 2$)$, where

$$
(\text { threshold } 2)=\sin \left(10^{\circ}\right) \text {. }
$$

Case 4: Case 1 but with $I_{3}, I_{4}>$ (threshold 1) and $I_{7}>$ (threshold 3), where

$$
(\text { threshold } 3)=\frac{0.3 \times\left|\xi_{4}+\eta_{4}\right|}{\sqrt{I_{1} I_{2}}} .
$$

(Threshold 1) is based on Groom-Bailey (1989) decomposition indicators of weak $2 \mathrm{D}$ behavior in the Alberta data. That is, a small difference in the phase along and perpendicular to the decomposed regional azimuth, coupled with small twist and shear identify a period at which two-dimensional behavior is observable. The weakest two-dimensional anomaly, near site 227 in southwest-Saskatchewan (shown on Fig. 1) and discussed in detail by Boerner et al. (1998, 2000), was used to calibrate (threshold 1). A special case of strong galvanic distortion of $1-$ or $2 \mathrm{D}$ structure exists when $\xi_{4}=0$ and $\xi_{1} \neq 0$. However, Groom-Bailey analysis confirms the 1- or 2D classification indicated by our invariant analysis. (Threshold 2) is based on the Mohr circle trigonometry, and is a measure from the Mohr circle analysis that reveals distortion geometrically as a deflection of the circle origin from the $Z_{x y}$ axis. (Threshold 3 ) is based on Bahr's phase sensitive skew as advocated by Weaver et al. (2000), and the value 0.3 is based on the advice of Bahr (1991).

With this classification, the four cases define four zones:

Case $1 \Rightarrow 1 \mathrm{D}$ regional structure.

Case $2 \Rightarrow 2 \mathrm{D}$ regional structure with a preferred strike direction.

Case $3 \Rightarrow 2 \mathrm{D}$ regional structure with distortion due to local structure.

Case $4 \Rightarrow 3$ D regional structure.

It should be noted that in this classification we have included both the small-scale and twist-only distortion cases in one case (Case 3). Furthermore, magnetic distortions are assumed to be negligible (Agarwal and Weaver, 2000).

\section{Dimensionality in the Alberta Data}

The MT data were acquired using the LIMS equipment developed by the Geological Survey of Canada and cover a period range of approximately 4-30,000 s. The equipment, its installation, the recording of the data and the determination of the MT tensor quantities are described by Boerner et al. (2000), together with a general description of the data characteristics.

An example of a result of the invariant dimensionality analysis for one period (213.3 s) is shown in Fig. 1 with the dimensionality according to the cases above for each site indicated. Geoelectric strike directions determined from Groom-Bailey (1989) decomposition for a period band around $300 \mathrm{~s}$ are shown for the 2D and 3D sites in this figure and illustrate the predominately NE-SW strike direction for the whole region. This figure shows the mainly $1 \mathrm{D}$ area to the southeast, a feature that has been clearly indicated earlier (Boerner et al., 1998). The Red Deer Conductor (RDC) (Boerner et al., 2000, figure 13) is revealed in this figure as locally $3 \mathrm{D}$ with surrounding regions of $2 \mathrm{D}$ with distortion. It is apparent that these structures are dominantly 2D with local 3D variations, and the 3D aspects are emphasized near the conductor because of the strong polarization of the electric field. An extensive 2D zone northwest of the RDC corresponds to the Buffalo Head, Chinchaga and Wabamun accreted domains (Craven et al. 2000), and dominates the central region of the study area for this period. A 3D/2D galvanic zone similar to the RDC is located to the northwest across the boundary between the Ksituan magmatic belt and the Archean Nova Province to the west (figure 2 of Boerner et al., 2000). Figure 2 shows the classification analysis for $1280 \mathrm{~s}$ period. The $3 \mathrm{D}$ character to the northwest that is apparent in the $213.3 \mathrm{~s}$ data is no longer indicated as $3 \mathrm{D}$ at $1280 \mathrm{~s}$, implying that the feature must be relatively shallow. The distribution of the sites indicating 3D at $1280 \mathrm{~s}$ in the region of the RDC differs from that previously (213.3 s), illustrating that the complexity of this anomalous zone increases with period, or alternatively that more remote sites now see the 3D structure.

Results for twelve periods between $5.3 \mathrm{~s}$ and $20480.2 \mathrm{~s}$ are shown in Fig. 3. The presence of the RDC is apparent at all periods, but the changes in the plots as period increases are striking. At short periods, the 1D nature of the data is evident (reflecting induction in the overlying Phanerozoic sediments) but the data exhibit increasingly more 3D character as period increases. This latter effect is consistent with the fact that the sounding response is from a zone of greater spatial extent at longer periods and is manifest by the centers of the Mohr circles generally located farther away from the $Z_{x y}$ axis as period increases. However, this is not a steady change, since at intermediate periods (note 640.0, 1280.0 and $2560.0 \mathrm{~s}$ ) the $3 \mathrm{D}$ character to the northwest is not evident. Therefore, the increase in $3 \mathrm{D}$ character with period may indicate more laterally complex geoelectrical structure at depth, or a fundamental limitation of distortion analysis in that the indication of $3 \mathrm{D}$ at depth is a result of the tensor elements having different skin depths.

\section{Conclusions}

The magnetotelluric tensor invariants as defined by Weaver et al. (2000) in terms of Mohr circles provide insight into the regional pattern of the dimensionality and so can be used to classify the regional geoelectric complexity. 
The results for the Western Canada Basin indicate that the basin is mainly $1 \mathrm{D}$ for short periods ( $\sim \mathrm{s})$, though the Red Deer Conductor is a strong perturbation within that structure. As period increases, 2D zones are indicated, some with minor galvanic distortion and there are limited $3 \mathrm{D}$ zones where the geoelectric structure is truly 3D. At long period, the invariant approach indicates extensive 3D character for the subsurface. This analysis may indicate true 3D structures, but could also represent a fundamental limitation of galvanic distortion analysis. Any frequency dependent analysis is uncertain when different elements of the tensor represent substantially different skin depths. In the Alberta data, $Z_{x y}$ is essentially sampling crustal structure, whereas the $Z_{y x}$ component at the same period is sampling the mantle (Boerner et al., 2000). The TE and TM modes are independent so that the regional impedances estimated by the two modes are not necessarily penetrating to or sampling the same depths. It is clear that tensor elements may sample different depths. Separate inversions of TE-only and TM-only modes can indicate structure at substantially different depths or when two different mode impedances are transformed to depth through skindepth calculations or by means of Niblett-Bostick transforms different depths can be indicated. The result of this difference in the penetration depth may be an invariant and phase sensitive skew that looks $3 \mathrm{D}$ at long periods. However, the mantle does not have to be 3D, but such an effect may be a by-product of a 2D or 3D crust. Any distortion model is limited in this way. Moreover, traditional distortion analysis is only formally correct for a surface scattering body. Most of our understanding of 3D models is based on inhomogeneities embedded in layered halfspaces and a 2D host severely distorts the "incident" field on a 3D body, thereby distorting the response of that body. Distortion analyses fail to account for the polarization effect on the $3 \mathrm{D}$ body by the incident field because it is generally assumed that the scattering body is at the surface whereas a buried 3D body deflects induced currents vertically and around a buried anomaly. The apparent $3 \mathrm{D}$ character at longer periods may thus be a limitation of the assumptions behind the distortion analyses. Nevertheless, analyses based on the invariants of the impedance ten- sor can still be a valuable aid to interpretation, in identifying zones where 1D and 2D inversions can be applied, and zones where $3 \mathrm{D}$ inversions are required.

Acknowledgments. This work was supported by the Lithoprobe Alberta Transect, the Geological Survey of Canada, the University of Alberta and the Natural Science and Engineering Research Council of Canada. The authors thank the reviewers, Malcolm Ingham and Hiroshi Munekane, for their careful reading of the manuscript and their suggestions. This is Lithoprobe contribution number 1303 and GSC contribution number 2002130.

\section{References}

Agarwal, A. K. and J. T. Weaver, Magnetic distortion of the magnetotelluric tensor: a numerical study, Earth Planets Space, 52, 347-353, 2000.

Bahr, K., Geological noise in magnetotelluric data: a classification of distortion types, Phys. Earth Planet. Inter., 66, 24-38, 1991.

Boerner, D. E., J. A. Craven, R. D. Kurtz, G. M. Ross, and F. W. Jones, The Great Falls Tectonic Zone: suture or intracontinental shear zone?, Can. J. Earth Sci., 35, 175-183, 1998.

Boerner, D. E., R. D. Kurtz, J. A. Craven, G. M. Ross, and F. W. Jones, A synthesis of electromagnetic studies in the Lithoprobe Alberta Basin Transect: constraints on Paleoproterozoic indentation tectonics, Can. J. Earth Sci., 37, 1509-1534, 2000.

Craven, J. A., D. E. Boerner, R. D. Kurtz, G. M. Ross, and F. W. Jones, Electrical anisotropy beneath the Western Canada Sedimentary Basin, 2000 (in preparation).

Groom, R. W. and R. C. Bailey, Decomposition of magnetotelluric impedance tensor in the presence of local three-dimensional galvanic distortion, J. Geophys. Res., 94, 1913-1925, 1989.

Lilley, F. E. M., Diagrams for magnetotelluric data, Geophysics, 41, 766$770,1976$.

Lilley, F. E. M., Magnetotelluric analysis using Mohr Circles, Geophysics, 58, 1498-1506, 1993a.

Lilley, F. E. M., Mohr circles in magnetotelluric interpretation (i) simple static shift; (ii) Bahr's analysis, J. Geomag. Geoelectr., 45, 833-839, $1993 b$.

Lilley, F. E. M. (Ted), Three-dimensionality of the BC87 magnetotelluric data set studied using Mohr Circles, J. Geomag. Geoelectr., 45, 1107$1113,1993 \mathrm{c}$.

Weaver, J. T., A. K. Agarwal, and F. E. M. Lilley, Characterization of the magnetotelluric tensor in terms of its invariants, Geophys. J. Int., 141, 321-336, 2000.

F. W. Jones (e-mail: wjones@phys.ualberta.ca), R. A. Munro, J. A. Craven, D. E. Boerner, R. D. Kurtz, and R. D. Sydora 